Review

\title{
Micronutrients and respiratory infections: the biological rationale and current state of clinical evaluation
}

\author{
Sam Rowe 1, 2, Patrick D Collins 1, Susan E Stacey 3, Anitra C Carr 4
}

1 Adult Critical Care Unit, Newham University Hospital, Barts Health NHS Trust, London, UK

2 Department of Clinical Sciences, Liverpool School of Tropical Medicine, Liverpool, UK

3 Brighton Sports Medicine, Brighton, UK

4 Nutrition in Medicine Research Group, University of Otago, Christchurch, New Zealand

Correspondence to: Sam Rowe (Sam.Rowe2@nhs.net)

\begin{abstract}
A range of nutrients has been studied or proposed for use in preventing respiratory tract infections and reducing severity of respiratory infections. This article gives a narrative review of the existing literature, biological rationales and current state of clinical evaluation for micronutrient therapies. The importance of vitamin A, the B vitamins, vitamin C, vitamin D, Eicosapentaenoic acid (EPA), vitamin E, selenium, zinc and a range of combined therapies are discussed, looking at their effects on reducing rates of infection, reducing severity of infection and improved recovery from infection. Further discussion regarding the level of evidence required for nutritional interventions is included.
\end{abstract}

Key words Antioxidants, Immunonutrition, Micronutrients, Nutrition, Pneumonia, Sepsis, Viral illness

Received: 12 December 2020; accepted following double-blind peer review: 25 January 2021

\section{Introduction}

It has long been suspected that nutritional status influences the susceptibility to respiratory infections. Immunonutrition has been used for cytokine modulation in several settings. Specific nutrients may attenuate the cytokine storm associated with severe infections (Calder et al, 2020). Although pan-supplementation of micronutrients has not yet been proven clinically, pharmacokinetics and subgroup analyses suggest that those with deficiency gain the most from nutritional interventions (Donnino et al, 2016; Lykkesfeldt, 2020). This narrative review focuses on specific nutrients known to have immunomodulatory roles in preventing and reducing severity of respiratory infections.

\section{Vitamin A and retinoids}

Vitamin A (retinol and retinal) is a lipid-soluble vitamin that is crucial for maintaining immune and humoral regulation, growth and development, vision and epithelial integrity.

Vitamin A deficiency is rare in developed countries, but common in lower and middle income countries. Meta-analysis of vitamin A supplementation in children with viral pneumonia was neutral with respect to mortality, but indicated faster recovery times in pooled analysis in lower and middle income countries (Brown and Roberts, 2007). 
Retinoids are molecules closely related to vitamin A that potentiate type 1 interferon (IFN-I)mediated antiviral responses. The oral compound retinoid LAU-7b (fenretinide) is being investigated for use in mitigating the pulmonary inflammatory response in cystic fibrosis (NCT03265288). Empirical supplementation may be warranted in the treatment of children and pregnant women with viral pneumonia or diarrhoeal illness in settings with a high baseline prevalence of deficiency, or in adults with malnutrition, as recommended by the European Society for Clinical Nutrition and Metabolism (Singer et al, 2019).

\section{The B vitamins}

The B vitamins are essential water-soluble vitamins that play a range of roles in health and disease including maintaining normal mitrochondrial function (Donnino et al, 2016).

Vitamin $\mathrm{B}_{1}$ (thiamine) deficiency is common in patients with sepsis (ranging between 20 and $70 \%$ by country and method of assessment; Donnino et al, 2016). The limited evidence from a pilot study and observational studies suggests a reduction in mortality and improved lactate clearance from supplementation in patients with sepsis and thiamine deficiency (Donnino et al, 2016). Severe thiamine deficiency may result in a range of clinical syndromes including peripheral neuropathy, Wernicke-Korsakoff syndrome and high output cardiac failure ('shoshin' beriberi). Those at risk of deficiency should receive parenteral supplementation (Singer et al, 2019).

Vitamin $\mathrm{B}_{2}$ (riboflavin) plays several roles in redox reactions. In animal models, high doses ameliorate nitric oxide and cytokine release without inhibiting host defences (Toyosawa et al, 2004). Riboflavin also decreases metabolism of vitamins $\mathrm{B}_{6}$ and $\mathrm{B}_{12}$ (Toyosawa et al, 2004).

Vitamin $\mathrm{B}_{3}$ (niacin) is essential for carbohydrate, fatty acid and protein synthesis and metabolism as part of the nicotinamide adenine dinucleotide and nicotinamide adenine dinucleotide phosphate coenzymes (Mousa and Mousa, 2020). Niacin deficiency results in the fatal disease pellagra (characterised by dermatitis, diarrhoea and dementia; Mousa and Mousa, 2020), which is now rare without malabsorption or isoniazid use. Additionally, nicotinamide may have antiviral, antibacterial and immunomodulating properties.

Vitamin $\mathrm{B}_{5}$ (pantothenic acid) is a precursor to coenzyme A whose deficiency causes reduced cortisol production, proinflammatory effects, mood changes and arthralgia with 'burning feet syndrome' (Gheita et al, 2020).

Vitamin $\mathrm{B}_{6}$ (pyridoxine, pyridoxamine and pyridoxal) is used as a co-factor in regulating cellular metabolism, scavenging reactive oxygen species and the synthesis of neurotransmitters and haem. Total body stores are low and deficiency can develop quickly in malabsorption states, with dialysis or diuresis, via medication interactions (such as hydrocortisone) and through inadequate nutrition. Deficiency is associated with a range of symptoms including irritability, depression, confusion, inflammation of the tongue and seizures. Deficiency is also associated with anaemias and hyperhomocysteinaemia (Spinneker et al, 2007).

Vitamin $\mathrm{B}_{12}$ (cobalamine) is essential for metabolic pathways in lipid, carbohydrate and protein metabolism. Deficiency may cause anaemia and severe neurological complications. Vitamin $\mathrm{B}_{12}$ deficiency is common in Intensive Care Unit (ICU) patients and particularly in those with alcoholism. A number of authorities recommend increase intake of vitamin $\mathrm{B}_{12}$ during critical illness (Romain et al, 2016).

As B vitamin deficiencies rarely occur in isolation, many commercially available preparations provide mixed B vitamins. In the UK, Pabrinex provides these with additional vitamin C. Benefits of supplementation appear to be in deficient patients and consensus guidelines suggest 
screening for risk of deficiency and providing supplementation in those at significant risk of deficiency (Singer et al, 2019).

\section{Vitamin C}

Vitamin C (ascorbate) is a water-soluble vitamin that plays a part in a plethora of biosynthetic and regulatory functions and rapidly becomes depleted in inflammatory states. Vitamin $\mathrm{C}$ appears to have beneficial effects on both innate and adaptive immunity. Observational research suggests that individuals who are deficient in vitamin $\mathrm{C}$ are at increased risk of respiratory infections (Hamer et al, $\underline{2009}$ ). Of note, vitamin $\mathrm{C}$ hypovitaminosis may be common in lower and middle income countries and is associated with socioeconomic deprivation in other settings (Hamer et al, 2009; Rowe and Carr, 2020).

A Cochrane review of vitamin $\mathrm{C}$ supplementation in the common cold showed that vitamin $\mathrm{C}$ did not reduce the rate of infections (in the general population), but supplementation reduced the duration and severity of symptoms (Hemilä and Chalker, 2013). In extreme conditions (athletes, skiers, military exercises), vitamin $\mathrm{C}$ supplements prevented the common cold with a pooled relative risk (RR) of 0.48 (95\% confidence intervals (CI) 0.35-0.64) (Hemilä and Chalker, 2013). Vitamin C has shown trends towards reduced duration of symptoms in students with influenza and in other viral illnesses (Gorton and Jarvis, 1999). Of note, most of the trials of vitamin C in this context used oral doses in populations with likely high vitamin $\mathrm{C}$ status (levels are likely to be saturated, ie $\sim 70 \mu \mathrm{mol} /$ litre when the intake is $\sim 200 \mathrm{mg} /$ day, which can be obtained by a healthy diet), and as highlighted in a review on the pharmacokinetics of vitamin $C$ this will greatly attenuate benefits as plasma levels are only likely to be significantly altered in individuals with suboptimal status (Lykkesfeldt, 2020).

Vitamin $\mathrm{C}$ acts as the body's first-line antioxidant and many patients are deficient at the point of ICU admission. Vitamin C can attenuate the formation of neutrophil extracellular traps which are implicated in organ failure, may maintain endothelial barriers and reduce formation of vascular microthrombosis. Additionally vitamin $\mathrm{C}$ is an important cofactor for the synthesis and/or optimal function of catecholamines, vasopressin and cortisol (Carr et al, 2017). High doses of vitamin C ( $>2 \mathrm{~g}$ a day) are required to normalise plasma levels in severe infections and, given its limited gastrointestinal absorption, this is likely to be best delivered intravenously (Lykkesfeldt, 2020).

Following the publication of a retrospective observational study by Marik et al (2017), and a number of small preceding studies, vitamin $\mathrm{C}$ became an area of great interest in critical care. Over 30 studies have been registered with vitamin $C$ as monotherapy or combined with other treatments such as hydrocortisone and thiamine. A number of meta-analyses have been completed on this and while a mortality benefit is not yet clear, results suggest reduced duration of mechanical ventilation and reduced requirements for critical care length of stay from vitamin C supplements (Hemilä and Chalker, 2020). The CITRIS-ALI study using $200 \mathrm{mg} / \mathrm{kg} /$ day of vitamin C showed shorter length of stay in the ICU and reduced mortality as secondary outcomes (Fowler et al, 2019). This was not replicated at lower doses in combination with steroids and thiamine in the VITAMINS trial (Fujii et al, 2020), the same combination showed a reduction in duration of shock in the ORANGE trial (27 \pm 22 vs $53 \pm 38$ hours, $P<0.001$ ) (Iglesias et al, 2020). There remains significant equipoise as to whether vitamin $\mathrm{C}$ alone or in combination with hydrocortisone results in a mortality benefit in sepsis, acute respiratory distress syndrome (ARDS) or pneumonia.

\section{Vitamin D}

Vitamin D exists in two main forms, vitamin $\mathrm{D}_{3}$ (cholecalciferol) and vitamin $\mathrm{D}_{2}$ (ergocalciferol). Apart from its role in bone metabolism, vitamin D is implicated in many immune 
interactions with deficiency common in the setting of chronic viral infections. Deficiency is associated with an increased risk of influenza and viral lower respiratory tract infections.

In a meta-analysis of 11321 participants, aged 0-95 years, vitamin D supplementation reduced the risk of acute respiratory tract infection among all participants (adjusted odds ratio (OR) $0.88,95 \%$ CI 0.81-0.96). Subgroup analysis showed enhanced benefits in patients with baseline 25 hydroxyvitamin D levels <25 nmol/litre (adjusted OR 0.30, 95\% CI 0.17-0.53) and in those receiving daily treatment (Martineau et al, 2017).

Vitamin D deficiency is associated with a myriad of chronic conditions such as diabetes and cardiovascular disorders which mirrors the susceptibility to COVID-19. Early evidence is promising for a role of vitamin D3 in the treatment of COVID-19 infection (Castillo et al, 2020).

The evidence suggests that low serum levels of 25-hydroxyvitamin $\mathrm{D}_{3}$ are also common in severe infections (Amrein et al, 2014). Treatment with vitamin D in subgroups with severe deficiency may result in a mortality benefit in patients with sepsis (Amrein et al, 2014). As the side-effect profile of supplementation is favourable, routine testing or treatment of patients at high risk of deficiency been advocated.

\section{Vitamin E}

Vitamin E (tocopherol and tocotrienol) is a lipid-soluble antioxidant that shows synergism with vitamin $\mathrm{C}$ and plays a number of roles in optimal immune function in health and disease. Human studies have shown decreased rates of re-admission in elderly Canadian patients discharged following admission with pneumonia (Neupane et al, 2010) and reduced incidence of pneumonia in elderly men taking vitamin E supplements (Meydani et al, 2004). Additionally, the evidence suggests a reduction in the rates of common cold in elderly nursing home residents (Meydani et al, 2004).

\section{Vitamin $\mathbf{K}_{1}$}

Vitamin $\mathrm{K}_{1}$ (phylloquinone) is well known for its role in coagulation and is particularly important in inhibiting excess formation of thrombin. Additionally vitamin $\mathrm{K}_{2}$ (menaquinone) is of importance in bone formation and has important interactions with vitamin D. Co-administration of vitamin $\mathrm{K}_{2}$ alongside vitamin D may increase absorption and use (Schwalfenberg, 2017).

\section{Minerals}

Zinc

Zinc is one of the most abundant metals in nature and is essential in many biological processes. It has a key role in the stabilisation of protein structures and is a cofactor in innumerable enzymatic reactions in a catalytic, structural or regulatory role.

Zinc plays pleiotropic roles in human health including regulation of clotting, wound healing, cognitive function, prostaglandin production, insulin secretion and regulation of immunity.

Notably, zinc absorption has been influenced by vitamin D status in animal models with deficiencies in the two correlated in some human cohorts. Although severe deficiency of zinc is rare in the developed world, moderate deficiency can be found among the elderly and those with chronic disease (Chasapis et al, 2012). 
Zinc is essential for antioxidant activity and immunity, modulating cytokine release, phagocytosis, and immunoglobulins. Lymphopaenia in zinc deficiency occurs as a result of increased apoptosis of thymic pre-T and pre-B cells, suggesting a key role for zinc in lymphocyte maturation.

Zinc deficiency has been linked in particular to increased risk of diarrhoeal and respiratory illnesses (Chasapis et al, 2012). Zinc-deficient mice suffer excessive damage from reactive oxygen species when exposed to lipopolysaccharide experimentally, an effect partially ameliorated by pretreatment with zinc. Furthermore, genome-wide association studies examining septic patients with fatal outcome have identified multiple pathways associated with zinc homeostasis as being linked to mortality (Heyland et al, 2008). Appropriate supplementation of zinc has been shown to reduce the risk of recurrent infections in those with impaired immunity (including in HIV, trisomy 21) (Chasapis et al, 2012).

Many host proteins which viruses interact with are zinc binding and there have been some postulated direct antiviral actions of zinc in in-vitro models. Clinically, a meta-analysis suggested that zinc lozenges may reduce the duration of common cold symptoms (Hemilä and Chalker, 2015).

Although these findings are intriguing, the diagnosis of zinc deficiency at the point of critical illness is fraught. As a negative acute phase reactant, low serum levels of zinc are not synonymous with underlying deficiency and may recover in convalescence.

\section{Selenium}

Selenium is another trace element essential for human health. Selenoproteins are involved in combating and regulating oxidative stress in virtually all tissues. In particular selenium is required for the actions of glutathione. Selenium also has a role in immunity, particularly in the normal development and function of lymphocytes and natural killer cells.

Low serum levels of selenium are commonly seen in patients in ICUs and correlates with risk of mortality (Li et al, 2019). It is particularly common in those with underlying impaired nutrition or chronic inflammation (including those with diabetes and atherosclerosis). The prevalence of selenium deficiency is regional, with depletion in soil common in Europe.

Selenium deficiency may be a specific risk factor for severe sequalae of viral infections. In murine models of influenza, selenium deficiency predisposes towards altered cellular and cytokine response. In human bronchial epithelial cells grown in a selenium-deficient culture there is reduced antioxidant activity and elevated IL-6 production in response to influenza exposure (Hoffmann and Berry, 2008). Girodon et al (1999) demonstrated improved an humoral response to influenza vaccination and reduced rate of respiratory tract infections among older adults supplemented with zinc and selenium. Selenium is also linked to clinical outcomes in the setting of those with HIV -1, with deficiency a risk factor for superadded infection, particularly mycobacterial infection (Hoffmann and Berry, 2008). Last, Keshan disease is a cardiomyopathy linked to coxsackie viral infections endemic to a band of Chinese provinces with poor selenium content in their soils which is almost entirely preventable through selenium supplementation.

Several randomised controlled trials (RCTs) have examined intravenous supplementation of selenium in critical illness. Although there were early signals of promise, notably the mortality signals seen in the 2007 Selenium in Intensive Care trial, the most recent meta-analyses do not suggest benefit in patients with sepsis ( $\mathrm{Li}$ et al, 2019) or in patients in the ICU in general. However, point estimates of effect have in general favoured selenium and the confidence intervals from metaanalyses do not exclude small but clinically significant benefits. The doses used in clinical trials have varied substantially but although the ideal posology is unknown, differential effects have not been seen when trials are clustered into high or low dose studies (Li et al, 2019). Not all studies have 
involved populations with baseline deficiency (notably in North American cohorts). Although a large number of trials have been carried out, most are small scale. Even SISPECT, the largest trial $(n=1089)$, was powered for an unrealistic $10 \%$ absolute reduction in mortality in sepsis (Li et al, 2019). Even if selenium supplementation is of no benefit whatsoever at the time of presentation with critical illness, deficient mineral status at baseline may still alter the course of patients with infection.

\section{Omega-3 fatty acids}

Linolenic acid and its derivatives (eicosapentaenoic acid and docosahexaenoic acid) are essential omega-3 fatty acids and play vital roles in health and development. The International Society for the Study of Fatty Acids and Lipids recommends a daily intake of $500 \mathrm{mg}$ of eicosapentaenoic acid and docosahexaenoic acid (ISSFAL 2004).

Increasing omega-3 levels reduces synthesis of cytokines, inflammatory eicosanoids and reactive oxygen species. Furthermore, anti-inflammatory specialised pro-resolving mediators rise with omega-3. In animal bacterial pneumonia models, specialised pro-resolving mediators decreased lipopolysaccharide-induced epithelial proinflammatory cytokine release but without sacrificing bacterial source control. They also hasten resolution of inflammation in experimental ARDS.

Nutritional trials involving omega-3 fatty acids with or without antioxidants have been used in multiple trials of patients with ARDS. A Cochrane review of heterogeneous treatments including eicosapentaenoic acid and docosahexaenoic acid for ARDS suggests potential reductions in duration of mechanical ventilation and ICU length of stay, along with improved oxygenation. Mortality at day 28 was reduced (RR $0.64,95 \%$ CI $0.49-0.84, n=466$ from six trials) and there was a trend towards decreased long-term mortality (longest period reported) RR $0.79(0.59-1.07) n=1015$ (from 10 RCTs) (Dushianthan et al, 2019).

\section{Combined antioxidant preparations}

Studies have looked at using combined enteral antioxidant preparations showing mixed results to date. Early clinical studies suggested that combined antioxidants may attenuate cytokine surges. While studies in patients in Brazil demonstrated significant physiological and mortality benefits, these results have not been replicated in settings where micronutrient deficiencies are rare (Dushianthan et al, 2019).

\section{Discussion and implications for further research and clinical care}

The precautions taken with novel pharmacological compounds may be overly cautious if applied to the supplementation of biologically essential trace elements and minerals. While the threshold for the evidence level required to begin treatment should arguably be lower, clinical trials are still needed to evaluate the ideal patient group, posology and timing for nutritional interventions in emerging infective diseases. Repeatedly, trials of micronutrient supplementation have shown benefit in patients who are deficient, but for many trace elements serum levels are influenced by the inflammatory response and turnaround times for testing may not be sufficient to help inform acute management. Supplementation of micronutrients to all is appealingly pragmatic given their low cost, (generally) wide margin of safety and biological plausible actions, yet in the setting of clinical trials may lead to reduced statistical power, especially in higher income countries where the prevalence of malnutrition is lower. A more targeted approach of empirical treatment for high-risk groups with universal screening may be preferred.

\section{Conclusions}


Good nutritional care must not be overlooking in the care of patients with respiratory infections and the population at large. Additional targeted nutritional interventions should be considered as described. A number of studies are ongoing but further research is urgently required, particularly in low-income settings, to guide treatment for pneumonia, sepsis, COVID-19 and inevitable future viral pandemics.

\section{Key points}

- Trials of micronutrient supplementation have shown promising benefits as adjuvant treatments in preventing and treating severe respiratory infections

- Benefits of supplementation are likely to be greatest in those with baseline deficiency

- Future trials should concentrate on patient groups with high rates of micronutrient deficiencies

\section{Conflicts of interest}

The authors declare that there are no conflicts of interest.

\section{Acknowledgements}

The authors would like to thank Dr S Stacey, Consultant in Cardiothoracic Anaesthesia and Intensive Care, Barts Health NHS Trust, London, UK, for his involvement in the concept, supervision and editing of this article and K Antunes, Nutritionalist/Dietician, National Heart and Lung Institute, Airway Diseases Section, Imperial College, London, UK, for her nutritional expertise and assistance in writing and editing the article.

\section{References}

Amrein K, Schnedl C, Holl A et al. Effect of high-dose vitamin D3 on hospital length of stay in critically ill patients with vitamin D deficiency: the VITdAL-ICU randomized clinical trial. JAMA. 2014;312(15):1520-1530. https://doi.org/10.1001/jama.2014.13204

Brown N, Roberts C. Vitamin A for acute respiratory infection in developing countries: a metaanalysis. Acta Paediatrica. 2007;93(11):1437-1442. https://doi.org/10.1111/j.16512227.2004.tb02625.x

Calder P, Carr A, Gombart A et al. Optimal nutritional status for a well-functioning immune system is an important factor to protect against viral infections. Nutrients. 2020;12(4):1181-1110. https://doi.org/10.3390/nu12041181

Carr AC, Rosengrave PC, Bayer S et al. Hypovitaminosis C and vitamin C deficiency in critically ill patients despite recommended enteral and parenteral intakes. Crit Care. 2017;21(1):1-10. https://doi.org/10.1186/s13054-017-1891-y

Castillo ME, Costa LME, Barrios JMV et al. Effect of calcifediol treatment and best available therapy versus best available therapy on intensive care unit admission and mortality among patients hospitalized for COVID-19: a pilot randomized clinical study. J Steroid Biochem Molecular Biol. 2020;203:105751. https://doi.org/10.1016/j.jsbmb.2020.105751

Chasapis CT, Loutsidou AC, Spiliopoulou CA et al. Zinc and human health: an update. Arch Toxicol. 2012;86(4):521-534. https://doi.org/10.1007/s00204-011-0775-1 
Donnino MW, Andersen LW, Chase M et al. Randomized, double-blind, placebo-controlled trial of thiamine as a metabolic resuscitator in septic shock: a pilot study. Crit Care Med. 2016;44(2):360367. https://doi.org/10.1097/CCM.0000000000001572

Dushianthan A, Cusack R, Burgess VA et al. Immunonutrition for acute respiratory distress syndrome (ARDS) in adults. Cochrane Database Syst Rev. 2019;1(1). https://doi.org/10.1002/14651858.CD012041.pub2

Fowler AA, Truwit JD, Hite RD et al. Effect of vitamin C Infusion on organ failure and biomarkers of inflammation and vascular injury in patients with sepsis and severe acute respiratory failure: the CITRIS-ALI randomized clinical trial. JAMA. 2019;322(13):1261-1270. https://doi.org/10.1001/jama.2019.11825

Fujii T, Luethi N, Young PJ et al. Effect of vitamin C, hydrocortisone, and thiamine vs hydrocortisone alone on time alive and free of vasopressor support among patients with septic shock: the VITAMINS randomized clinical trial. JAMA. 2020;323(5):423-431. https://doi.org/10.1001/jama.2019.22176

Gheita AA, Gheita TA, Kenawy SA et al. The potential role of B5: a stitch in time and switch in cytokine. Phytother Res. 2020;34(2):306-314. https://doi.org/10.1002/ptr.6537

Girodon F, Galan P, Monget AL et al. Impact of trace elements and vitamin supplementation on immunity and infections in institutionalized elderly patients: a randomized controlled trial. Arch Intern Med. 1999;159(7):748-754. https://doi.org/10.1001/archinte.159.7.748

Gorton HC, Jarvis K. The effectiveness of vitamin C in preventing and relieving the symptoms of virus-induced respiratory infections. J Manipulative Physiol Therapeut. 1999;22(8):530-533. https://doi.org/10.1016/S0161-4754(99)70005-9

Hamer DH, Sempértegui F, Estrella B et al. Micronutrient deficiencies are associated with impaired immune response and higher burden of respiratory infections in elderly Ecuadorians. J Nutr. 2009;139(1):113-119. https://doi.org/10.3945/jn.108.095091

Hemilä H, Chalker E. Vitamin C for preventing and treating the common cold. Cochrane Database Syst Rev. 2013;31(1). https://doi.org/10.1002/14651858.CD000980.pub4

Hemilä $\mathrm{H}$, Chalker $\mathrm{E}$. The effectiveness of high dose zinc acetate lozenges on various common cold symptoms: a meta-analysis. BMC Fam Pract. 2015;16(1):24. https://doi.org/10.1186/s12875-0150237-6

Hemilä $\mathrm{H}$, Chalker E. Vitamin $\mathrm{C}$ may reduce the duration of mechanical ventilation in critically ill patients: a meta-regression analysis. J Intensive Care. 2020;8(1):15. https://doi.org/10.1186/s40560020-0432-y

Heyland DK, Jones N, Cvijanovich NZ et al. Zinc supplementation in critically Ill patients: a key pharmaconutrient? J Parenter Enteral Nutr. 2008;32(5):509-519. 10.1177/0148607108322402

Hoffmann PR, Berry MJ. The influence of selenium on immune responses. Mol Nutr Food Res. 2008;52(11):1273-1280. https://doi.org/10.1002/mnfr.200700330

Iglesias J, Vassallo AV, Patel VV et al. Outcomes of metabolic resuscitation using ascorbic acid, thiamine, and glucocorticoids in the early treatment of sepsis. Chest. 2020;158(1):164-173. https://doi.org/10.1016/j.chest.2020.02.049 
ISSFAL International Society for the Study of Fatty Acids and Lipids 2004. Report of the SubCommittee on recommendations for intake of polyunsaturated fatty acids in healthy adults(internet). (cited 28/03/2021). Available from https://www.issfal.org/statement-3

Li S, Tang T, Guo P et al. A meta-analysis of randomized controlled trials: efficacy of selenium treatment for sepsis. Medicine. 2019;98(9):e14733. https://doi.org/10.1097/MD.0000000000014733

Lykkesfeldt J. Redox biology on the effect of vitamin C intake on human health: how to (mis) interprete the clinical evidence. Redox Biol. 2020;34:101532.

https://doi.org/10.1016/j.redox.2020.101532

Marik PE, Khangoora V, Rivera R et al. Hydrocortisone, vitamin C, and thiamine for the treatment of severe sepsis and septic shock. Chest. 2017;151(6):1229-1238.

https://doi.org/10.1016/j.chest.2016.11.036

Martineau AR, Jolliffe DA, Hooper RL et al. Vitamin D supplementation to prevent acute respiratory tract infections: systematic review and meta-analysis of individual participant data. BMJ (Online). 2017;356:65-83. https://doi.org/10.1136/bmj.i6583

Meydani SN, Han SN, Hamer DH et al. Vitamin E and respiratory infection in the elderly. Ann New York Acad Sci. 2004;1031(1):214-222. https://doi.org/10.1196/annals.1331.021

Mousa TY, Mousa OY. Nicotinic acid deficiency. 2020.

https://www.ncbi.nlm.nih.gov/books/NBK557749 (accessed 18 March 2021)

Neupane B, Walter SD, Krueger P et al. Predictors of inhospital mortality and re-hospitalization in older adults with community-acquired pneumonia: a prospective cohort study. BMC Geriatr. 2010;10(1):22. https://doi.org/10.1186/1471-2318-10-22

Romain M, Sviri S, Linton DM et al. The role of vitamin B12 in the critically ill: a review. Anaesthesia Intens Care. 2016;44(4):447-452. https://doi.org/10.1177/0310057X1604400410

Rowe S, Carr AC. Global vitamin C status and prevalence of deficiency: a cause for concern? Nutrients. 2020;12(7):2008. https://doi.org/10.3390/nu12072008

Schwalfenberg GK. Vitamins K1 and K2: the emerging group of vitamins required for human health. J Nutr Metab. 2017;2017:1-6. https://doi.org/10.1155/2017/6254836

Singer P, Blaser AR, Berger MM et al. ESPEN Guideline ESPEN guideline on clinical nutrition in the intensive care unit. Clin Nutr. 2019;38(1):48-79. https://doi.org/10.1016/j.clnu.2018.08.037

Spinneker A, Sola R, Lemmen V et al. Vitamin B6 status, deficiency and its consequences-an overview. Nutr Hosp. 2007;22(1):7-24

Toyosawa T, Suzuki M, Kodama K et al. Highly purified vitamin B2 presents a promising therapeutic strategy for sepsis and septic shock. Infect Immun. 2004;72(3):1820-1823.

https://doi.org/10.1128/IAI.72.3.1820-1823.2004 